\title{
Estrategias educativas inclusivas y su relación con la autoeficacia de docentes en formación
}

Inclusive educational strategies and their relationship with the selfemployment of teachers in training

\author{
Volumen 20, Número 1 \\ Enero - Abril \\ pp. 1-25
}

\author{
Luisa Dolores Murillo Parra \\ Dora Yolanda Ramos Estrada \\ Ismael García Cedillo \\ Mirsha Alicia Sotelo Castillo
}

Citar este documento según modelo APA

Murillo Parra, Luisa Dolores; Ramos Estrada, Dora Yolanda; García Cedillo, Ismael y Sotelo Castillo, Mirsha Alicia. (2020). Estrategias educativas inclusivas y su relación con la autoeficacia de docentes en formación. Revista Actualidades Investigativas en Educación, 20(1), 1-25. Doi. 10.15517/aie.v20i1.40060 


\title{
Estrategias educativas inclusivas y su relación con la autoeficacia de docentes en formación
}

Inclusive educational strategies and their relationship with the self-employment of teachers in training

\author{
Luisa Dolores Murillo Parra ${ }^{1}$ \\ Dora Yolanda Ramos Estrada² \\ Ismael García Cedillo 3 \\ Mirsha Alicia Sotelo Castillo
}

\begin{abstract}
Resumen: El objetivo del artículo fue analizar la relación entre las estrategias educativas inclusivas y la autoeficacia de docentes en formación de escuelas normales, así como realizar una comparación entre escuela normal urbana y escuela rural. El enfoque utilizado fue cuantitativo con un alcance correlacional comparativo. Participaron 166 docentes en formación de Licenciatura en Educación Primaria de dos Escuelas Normales del Sur de Sonora, México, en 2018. Se aplicaron dos instrumentos: Estrategias para fortalecer el aprendizaje: Cuestionario para el profesor, de Mitchell, y la Escala de Autoeficacia para Implementar Prácticas Inclusivas de Sharma, Loreman y Forlin. Los resultados mostraron que la autoeficacia presenta relación significativa con las estrategias inclusivas de las personas docentes en formación. Se encontró que el estudiantado de escuela normal urbana presenta mayores estrategias educativas inclusivas que el de la escuela normal rural. Se destacó la necesidad de formación y capacitación en educación inclusiva, así como mayores recursos materiales y personales para trabajar con estudiantes con necesidades educativas especiales. Es importante que, durante la formación docente, en las escuelas normales urbanas y rurales, se dote a las personas docentes en formación de preparación, herramientas, conocimientos, así como motivación y espacios de práctica adecuados para poder emprender el rol que les corresponde en un contexto inclusivo.
\end{abstract}

Palabras clave: educación inclusiva, estrategias educativas, autoeficacia, formación docente.

Abstract: The objective of the article was to analyze the relationship between inclusive educational strategies and the self-efficacy of teachers in the formation of normal schools, as well as make a comparison between normal urban and rural schools. The approach used was quantitative with a comparative correlational scope, 166 students of Bachelor of Primary Education from two Normal Schools of South Sonora, Mexico participated in 2018. Two instruments were applied: Strategies to strengthen learning: Questionnaire for the teacher, Mitchell and the Selfefficacy Scale to Implement Inclusive Practices of Sharma, Loreman and Forlin. The results showed that selfefficacy presents a significant relationship with the inclusive strategies of teachers in training. It was found that urban normal school students have greater inclusive educational strategies than rural normal school students. The need for training and education in inclusive education was highlighted, as well as greater material and personal resources to work with students with special educational needs. It is important that, during teacher training, in urban and rural normal schools, teachers are given training in preparation, tools, knowledge, as well as motivation and appropriate practice spaces to undertake their rightful role in an inclusive context.

Keywords: inclusive education, educational strategies, self-efficacy, teacher training. 1 Asistente Académico Administrativo del Instituto Tecnológico de Sonora, en el programa educativo
Licenciado en Educación Infantil en Navojoa, Sonora, México. Dirección electrónica: luisadmp@hotmail.com ORCID https://orcid.org/0000-0002-6589-9980

2 Profesora investigadora de tiempo completo en el Instituto Tecnológico de Sonora, Departamento de Psicología en Obregón, Sonora, México. Dirección electrónica: dramos@itson.edu.mx ORCID https://orcid.org/0000-0001-7933-3753

${ }^{3}$ Profesor-Investigador de la Universidad Autónoma de San Luis Potosí, Facultad de Psicología, México. Dirección electrónica: garcia.ismael52@gmail.com ORCID https://orcid.org/0000-0002-4820-5633

${ }^{4}$ Profesora investigadora de tiempo completo en el Instituto Tecnológico de Sonora, Departamento de Psicología en Obregón, Sonora, México.Dirección electrónica: mirsha.sotelo@itson.edu.mx ORCID https://orcid.org/0000-0001-9838-189X

Artículo recibido: 30 de junio, 2019

Enviado a corrección: 25 de octubre, 2019

Aprobado: 18 de noviembre, 2019 


\section{Introducción}

La educación inclusiva en la actualidad es considerada un derecho humano y de justicia social que promueve el respeto, la aceptación de las diferencias y la igualdad de oportunidades. Al mismo tiempo, valora la diversidad y busca desarrollar estrategias que favorezcan el proceso de enseñanza aprendizaje, con el fin de disminuir la desigualdad y exclusión en el contexto educativo (Chiner, 2011; Serrato Almendarez y García Cedillo, 2014). La educación inclusiva impulsa el desarrollo de una educación de calidad gracias al uso adecuado de los recursos, además de la colaboración y el compromiso de las instituciones formadoras de docentes, la comunidad escolar, los directivos, padres de familia y el profesorado. Estos últimos son considerados una pieza fundamental en el desarrollo intelectual, moral y afectivo de niños, adolescentes y jóvenes, por la realización de acciones pedagógicas pertinentes para lograr objetivos y facilitar el proceso de educación inclusiva (Flores y Villardón, 2015; García Cedillo y Romero Contreras, 2016; Instituto Nacional para la Evaluación de la Educación [INEE], 2015).

Durante la formación inicial, el profesorado debe adquirir las competencias específicas para el desarrollo de prácticas educativas inclusivas. Fernández Batanero y Hernández Fernández (2013), así como Ortega y Ortiz (2015) mencionan que las prácticas educativas inclusivas son las estrategias que lleva a cabo el profesorado con el fin de adaptarse y de atender la diversidad de culturas, preferencias, estilos de aprendizaje, intereses, entre otros aspectos. Asimismo, las prácticas educativas inclusivas permiten nuevas formas de trabajo en los centros escolares y la eliminación de barreras para el aprendizaje y la participación.

Algunas de las estrategias citadas por Fernández (2014) para un contexto inclusivo son: la identificación de necesidades, el fomento del trabajo cooperativo, la adaptación de tecnología, la diversificación de contenidos, materiales y recursos, estrategias relacionadas con el clima del aula, motivación, entre otras. Beltrán (2004) considera que estas estrategias deben ir enfocadas a promover el aprendizaje significativo, la autonomía y autorregulación del estudiantado. Esto representa un desafío a las instituciones formadoras de docentes.

En México, las principales instituciones formadoras de docentes son las Escuelas Normales urbanas y rurales, las cuales son reguladas por la Secretaría de Educación Pública (SEP) en cuanto a sus políticas de formación para el nivel de educación básica (Infante, 2010; Navarrete, 2015). Estas instituciones deben proporcionar conocimientos sobre el currículo, además de espacios de práctica para el personal docente en formación, en donde 
se adquiera el dominio de las metodologías y estrategias más adecuadas para el proceso de enseñanza aprendizaje de todo el alumnado, con o sin discapacidad (Durán y Giné, 2011). Lo anterior se relaciona con un aspecto de la docencia que es la autoeficacia, la cual se refiere a las creencias que tiene una persona de poseer las capacidades necesarias para realizar alguna acción o actividad, e involucra aspectos cognitivos, motivacionales y afectivos (Bandura, 1977).

Se dice que el profesorado con baja autoeficacia suele sentirse desmotivado y poner poco esfuerzo en sus tareas, ya que considera que no cuenta con las habilidades necesarias para lograr el pleno desarrollo del alumnado. Por el contrario, el profesorado con un sentido de alta autoeficacia se encuentra motivado, hace esfuerzos constantes, es innovador y persistente. Por ello, la autoeficacia se considera como un elemento clave para el esfuerzo, la persistencia y la autodeterminación del profesorado, de lo contrario, podrían tener expectativas negativas, lo que puede provocar el fracaso en la implementación de prácticas educativas inclusivas (Adame, 2015; Lamorey y Wilcox, 2005).

La Organización de las Naciones Unidas (ONU), en 2007, señaló que el profesorado manifestó no estar lo suficientemente preparado para implementar la educación inclusiva en el alumnado con Necesidades Educativas Especiales (NEE). Asimismo, en el Estudio Internacional sobre la Enseñanza y el Aprendizaje (TALIS) del 2013, se indicó que entre el año 2008 y 2013 aumentó el porcentaje de profesores mexicanos que mencionaron la necesidad de cursos para la enseñanza al alumnado con NEE y cursos para la enseñanza multicultural (Backhoff Escudero y Pérez Moran, 2015).

Romero Contreras y García Cedillo (2013) señalan que, en la actualidad, la formación docente no proporciona los recursos suficientes para que el personal docente en formación lleve a cabo, de manera oportuna, sus prácticas, ya que solo proporciona los contenidos que deben enseñar, pero no se les instruye cómo deben hacerlo y/o adaptar esos contenidos a las necesidades del alumnado. En este aspecto, se ha informado que solo el $50 \%$ de las personas egresadas de Escuelas Normales, a nivel nacional, ha resultado idóneo para ingresar al Servicio Profesional Docente (Mendoza, 2015).

En varios trabajos se ha encontrado una escasa presencia de estrategias para la atención a la diversidad, metodologías poco flexibles y escaso entendimiento de la educación inclusiva (Darretxe Urrotxi, Goikoetxea Piedrola y Fernández González, 2013; Ortega Tello y Ortiz Ochoa, 2015; Ramírez García y Muñoz Fernández, 2012). Otros autores 
han resaltado la importancia de realizar investigaciones que incidan en el personal docente en formación de las Escuelas Normales, de manera que puedan analizarse sus perspectivas y aspectos afectivos, ya que este será el encargado de transmitirlas en un futuro al alumnado de educación básica (Novo-Corti, Muñoz-Cantero y Calvo-Babio, 2015; Rubio-Moreno, Castro-López y Félix-Salazar, 2015; Tárraga, Peirats y Grau, 2013; Vélez, 2013).

\section{Referente teórico}

Las prácticas educativas inclusivas hacen referencia a proporcionar programas de calidad educativa para cualquier estudiante, pues implican la implementación de estrategias enfocadas en la participación, progreso y logro de cada uno. Toda práctica inclusiva tiene dos características principales: 1) se trabaja con grupos diversos y 2) se cuenta con el apoyo de otros profesionales y recursos materiales apropiados, con la finalidad de beneficiar al alumnado durante su proceso de aprendizaje (Muntaner Guasp, Rosselló Ramón y De la Iglesia Mayol, 2016). Existen varias investigaciones que han estudiado esta temática, Lledó Carreres y Arnaiz Sánchez (2010) identificaron en las prácticas del profesorado, algunos ejemplos de adaptaciones curriculares. Sin embargo, las personas participantes manifestaron la necesidad de renovar las estrategias de enseñanza debido a que contaban con poca preparación, escasez de recursos y falta de apoyo de otros profesionales para atender la diversidad.

Por su parte, Fernández Batanero (2013) utilizó la metodología cualitativa para conocer la percepción del profesorado sobre sus propias competencias en educación inclusiva y encontró que comparte una visión positiva de la inclusión, además de proporcionar un espacio de tutorías, buena comunicación, metodologías alternativas, adecuación de materiales/actividades y una adecuada atención a la familia. Por el contrario, otros estudios han indicado que el profesorado ha sido poco flexible en cuanto a los materiales y metodologías que utiliza diariamente en su labor. Se ha mostrado poca sensibilidad respecto a la diversidad cultural del alumnado, así como un bajo nivel de apoyo para el aprendizaje y la participación, por lo que recomiendan analizar la práctica del profesorado y las concepciones hacia la educación inclusiva en conjunto (Darretxe Urrotxi et al., 2013; Ortega Tello y Ortiz Ochoa, 2015).

Atendiendo a este aspecto, en Chile, Santos Arrieta (2014) identificó la percepción del profesorado sobre la educación inclusiva y la relación con sus prácticas educativas 
inclusivas. Se encontraron resultados positivos con respecto a las percepciones, sin embargo, se resaltó el aspecto de la poca formación, el tiempo limitado y el escaso apoyo recibido. En relación con las prácticas, se concluyó que hace falta mejorar el aspecto de estrategias de agrupamiento de alumnos y adaptación de las actividades.

Flores Barrera y García Cedillo (2016) identificaron que, dentro de las principales necesidades del alumnado con discapacidad en el aula regular, se encuentran la flexibilidad del profesorado y la implementación de adecuaciones curriculares y ajustes metodológicos, ya que la capacitación recibida ha sido en su mayoría teórica, por lo que no se ha logrado una sensibilización e impacto en sus prácticas docentes.

Flores Barrera, García Cedillo y Romero Contreras (2017) encontraron que las personas formadoras de docentes de escuelas normales llevan a cabo estrategias relacionadas con el uso del tiempo, evaluación, reflexión y sensibilización. Sin embargo, carecen de apoyos en el aula para poner en juego una metodología adecuada, estrategias relacionadas con el vínculo docente-estudiante y escaso conocimiento sobre educación inclusiva.

Enríquez Félix (2015) llevó a cabo un estudio en la única Escuela Normal Rural del Estado de Sonora, la cual es la participante en este trabajo y encontró que los formadores de docentes tienden a realizar estrategias conductuales, tales como manejo de la disciplina, fomento de las relaciones interpersonales y evaluación del aprendizaje. Sin embargo, se resalta la importancia de contar con elementos pedagógicos y tener un actuar constructivista en el que la persona docente en formación pueda ser un agente activo de su propio aprendizaje. Asimismo, Civera (2015) menciona que la información disponible de las escuelas normales rurales de los años 70 hasta la actualidad proviene, la gran mayoría, de fuentes periodísticas en las que destacan conflictos entre estas y las autoridades. No obstante, se muestra poco sobre los motivos de los conflictos, así como lo que sucede en la vida cotidiana de las escuelas.

En cuanto a la autoeficacia, esta fue introducida por Albert Bandura (1977) y se refiere a las creencias que tiene una persona de poseer las capacidades necesarias para realizar alguna acción o actividad, involucra aspectos cognitivos, motivacionales y afectivos. Según Covarrubias Apablaza y Mendoza Lira (2013), la autoeficacia docente consiste en las creencias individuales que tiene el profesorado sobre sus habilidades y capacidades para la enseñanza, así como para planificar y desarrollar actividades que promuevan el aprendizaje. 
Lynn Peebles (2012) analizó el impacto de un curso de educación inclusiva y experiencia de campo en la autoeficacia de docentes en formación. Se concluyó que el curso y la experiencia de campo incrementaron significativamente la autoeficacia de las personas participantes. Se destacó que aquellos docentes en formación que ya tenían experiencia previa trabajando con el alumnado con NEE presentaron niveles altos de autoeficacia durante todo el estudio.

Loreman, Sharma y Forlin (2013) realizaron un estudio comparativo en Canadá, Australia, Hong Kong e Indonesia sobre la autoeficacia que presentaban docentes en formación y concluyeron que existían diferencias significativas en los cuatro contextos. Algunos de los factores que explicaron estas diferencias son: los tipos de programa para el profesorado, las leyes que se tienen sobre inclusión, el contacto previo que se ha tenido con personas con discapacidad y si se tiene o no experiencia trabajando con este tipo de alumnado.

Romero Contreras, García Cedillo, Forlin y Lomelí Hernández (2013) mencionan que el nivel de capacitación y conocimiento de políticas inclusivas, así como la experiencia y confianza en la enseñanza del alumnado con discapacidad o NEE pueden incidir en la autoeficacia del profesorado. Por lo tanto, la formación docente debe enfocarse en el desarrollo de niveles altos de autoeficacia hacia la educación inclusiva, de manera que puedan enfrentar el reto de enseñar al alumnado con NEE y promover su aceptación e inclusión en escuelas regulares. A su vez, los formadores de docentes de escuelas normales deben garantizar la supervisión y asesoramiento al cuerpo docente en formación durante su preparación (Forlin, García Cedillo, Romero Contreras, Fletcher y Rodríguez Hernández, 2010).

En ese sentido y tomando en cuenta la revisión de la literatura, en el presente estudio se plantea como objetivo analizar la relación entre las estrategias educativas inclusivas y la autoeficacia de docentes en formación de Escuelas Normales, así como realizar una comparación entre escuela normal urbana y rural.

\section{Metodología}

\subsection{Enfoque}

El estudio corresponde al enfoque cuantitativo con un diseño no experimental de alcance correlacional comparativo, dado que se busca conocer la relación entre variables, 
así como comparar los resultados por el tipo de escuela de las personas participantes (Hernández Sampieri, Fernández Collado y Baptista Lucio, 2014).

\subsection{Población de estudio}

En el estudio participaron 166 docentes en formación de instituciones de formación docente de la región del Sur de Sonora en el año 2018, quienes se encontraban inscritos en la Licenciatura en Educación Primaria. Participaron dos Escuelas Normales públicas federales: 1) El Centro Regional de Educación Normal (CREN) "Rafael Ramírez Castañeda" ubicado en la ciudad de Navojoa, Sonora, México, que ofrece licenciaturas escolarizadas de 8 semestres en educación preescolar, educación primaria, educación preescolar intercultural bilingüe y educación primaria intercultural bilingüe; 2) La Escuela Normal Rural (ENR) "Gral. Plutarco Elías Calles", ubicada en el municipio de Etchojoa, Sonora a una distancia aproximada de $29 \mathrm{~km}$ de la ciudad de Navojoa, Sonora, la cual únicamente ofrece la licenciatura en educación primaria. Por ello, se procuró que la población fuera equivalente y solamente se consideró esa licenciatura para la investigación. Medrano Camacho, Ángeles Méndez y Morales Hernández (2017) reportaron en el Instituto Nacional para la Evaluación de la Educación (INEE) que en el año 2016 la matrícula de ingreso en educación normal en el estado de Sonora fue de 3328, egresados 912 y titulados 891.

En este estudio, el $22 \%$ de las personas docentes en formación fueron de sexto semestre y el $77 \%$ de octavo. Los participantes realizaban prácticas profesionales y tenían experiencia frente a grupo lo cual fue uno de los requisitos para participar en el estudio. El $53 \%(n=89)$ fue de sexo femenino y el 46\% ( $n=77)$ masculino, con una media de edad de 22 años ( $D E=.89$ ). El $53.6 \%$ de los docentes en formación correspondía a escuela normal rural (ENR) y el 46.4\% a escuela normal urbana (CREN). Para la selección de la población de estudio se optó por un muestreo no probabilístico por conveniencia.

\subsection{Técnicas de recolección}

El primer instrumento empleado fue el cuestionario de Estrategias para fortalecer el aprendizaje: Cuestionario para el profesor de Mitchell (2014), traducido y adaptado para la población mexicana por Fletcher, García y Romero en 2015. El propósito de este cuestionario es identificar las prácticas educativas inclusivas en el contexto escolar y puede ser empleado tanto para el profesorado como para docentes en formación. 
El instrumento se compone por 34 reactivos organizados en cinco subescalas: 1) contexto de aprendizaje, 2) estrategias de enseñanza, 3) estrategias del comportamiento, 4) estrategias de cooperación interinstitucional y 5) estrategias de recursos para el aprendizaje. Se utiliza una escala Likert de 4 opciones de respuesta: muy a menudo/muchas veces (calificación de 4); a menudo (3); de vez en cuando/pocas veces (2); rara vez/nunca (1). El índice de confiabilidad de alpha de Cronbach para la escala total fue de 0,89 , considerado un nivel adecuado (George y Mallery, 2003).

El segundo cuestionario fue la Escala de Autoeficacia para Implementar Prácticas Inclusivas de Sharma, Loreman y Forlin (2011), traducida al español y adaptada por Forlin et al. (2009). Este cuestionario identifica las percepciones de autoeficacia para llevar a cabo la educación inclusiva y puede ser empleado tanto para el profesorado como para docentes en formación.

El instrumento consta de 18 reactivos organizados en tres dimensiones: 1) eficacia para dar instrucciones inclusivas, referida a las estrategias que se llevan a cabo para promover la inclusión del alumnado; 2) eficacia en la colaboración, que hace referencia a las percepciones individuales de la eficacia del profesorado en el trabajo con los padres y otros profesionales; y 3) eficacia en el manejo del comportamiento, relacionada con la eficacia del profesorado para manejar comportamientos disruptivos en el grupo. Se utiliza una escala Likert de 4 opciones de respuesta: totalmente de acuerdo (4); de acuerdo (3); en desacuerdo (2); totalmente en desacuerdo (1). El valor de alpha de Cronbach para la escala total fue 0,91, que indica un nivel adecuado de confiabilidad (George y Mallery, 2003).

\subsection{Procedimiento de recolección de datos}

En primer lugar, se solicitó autorización a los subdirectores académicos de las dos Escuelas Normales y se les comunicó los objetivos del trabajo, así como la metodología a seguir para la aplicación de los cuestionarios. Posteriormente, se acordaron las fechas en las que se podría asistir, el horario y los espacios disponibles en los que se llevaría a cabo la aplicación. La aplicación de cuestionarios se realizó en las aulas de clase de las dos instituciones educativas. Se les explicó a las personas participantes las instrucciones para responder y se les informó sobre la confidencialidad de la información. Al comprobar que los cuestionarios no representan ningún daño para la salud, se optó por el consentimiento 
informado de manera verbal. La aplicación de instrumentos tuvo una duración aproximada de 30 minutos.

\subsection{Procesamiento de análisis}

Para el análisis de la información se realizó la captura de la información en el paquete estadístico SPSS (Statistical Package for the Social Sciences) versión 23. Con el objetivo de analizar las estrategias educativas inclusivas y la autoeficacia de docentes en formación de escuelas normales, se realizaron análisis descriptivos de medias. La comparación de medias se realizó según la ubicación de la escuela en la que estudiaban (urbana y rural), se llevó a cabo con la prueba $\mathrm{T}$ de Student para muestras independientes. Para conocer la relación entre las variables mencionadas se procedió a realizar la correlación de Pearson. El análisis de normalidad de la población se verificó con la prueba Kolmogorov-Smirnov la cual indicó valores mayores a 0.05 . Se estable un nivel de significancia del $5 \%$.

\section{Resultados}

Para dar respuesta al objetivo planteado, en primer lugar, se analizaron las respuestas del cuerpo docente en formación en los diferentes reactivos del Cuestionario de Estrategias para Fortalecer el Aprendizaje. Se obtuvo una media general de 3.26, lo que indica predisposiciones positivas para desarrollar estrategias inclusivas (ver Tabla 1).

En la primera subescala contexto de aprendizaje, el cuerpo docente en formación mencionó que en su práctica trata de crear un ambiente en el aula seguro, predecible y motivador e indicó que la escuela normal a la que pertenece reconoce el derecho del alumnado con NEE a ser educado en salones regulares. Asimismo, señaló utilizar estrategias de trabajo colaborativo entre alumnado con distintos niveles de habilidad.

En la subescala estrategias de enseñanza, las personas docentes en formación mencionaron que animan al alumnado a establecer sus propias metas y evaluar continuamente su progreso para alcanzarlas. También indicaron que revisan y practican en sus clases las principales ideas de las lecciones anteriores.

En la subsescala estrategias del comportamiento, las personas docentes en formación mencionaron que enseñan habilidades sociales adecuadas al alumnado. La media más baja se presentó en el reactivo: "Mi escuela tiene programas de apoyo conductual positivo que enfatizan la prevención y la reducción de problemas de conducta crónicos, la enseñanza 
activa de habilidades adaptativas, un continuo de consecuencias para los problemas de conducta e intervención".

Respecto a la subescala de estrategias de cooperación interinstitucional, las personas docentes en formación indicaron que tratan de asegurarse de que el alumnado tenga suficiente tiempo para aprender, así como proporcionar retroalimentación a todos.

En la subescala recursos para el aprendizaje se encontró que las personas docentes en formación utilizan las tecnologías de información y comunicación disponibles. Asimismo, señalaron que los accesos en salones de clase y en la escuela normal son apropiados para personas con discapacidad. Las medias más bajas se encontraron en los reactivos relacionados con los apoyos que se reciben en las instituciones y en el equipamiento de personal especializado.

Tabla 1

Puntuaciones medias del Cuestionario de Estrategias para Fortalecer el Aprendizaje en personas docentes en formación del Sur de Sonora, México, 2018

\begin{tabular}{lcc}
\hline \multirow{2}{*}{ Subescalas } & \multicolumn{2}{c}{$\begin{array}{c}\text { Docentes en } \\
\text { formación }\end{array}$} \\
\cline { 2 - 3 } & $\mathrm{M}$ & $\mathrm{DE}$ \\
\hline 1. Contexto de aprendizaje & 3.32 & 0.36 \\
2. Estrategias de enseñanza & 3.33 & 0.43 \\
3. Estrategias del comportamiento & 3.21 & 0.48 \\
4. Estrategias de cooperación interinstitucional & 3.36 & 0.42 \\
5. Recursos para el aprendizaje & 2.81 & 0.71 \\
\hline Total & 3.26 & 0.34 \\
\hline
\end{tabular}

Fuente: Elaboración propia, 2019

De manera general, se destacó que las principales estrategias educativas inclusivas llevadas a cabo por las personas docentes en formación de las escuelas normales fueron el trabajo colaborativo, la enseñanza de habilidades sociales, el uso de las tecnologías de información y comunicación (TIC) en el aula, y la retroalimentación al alumnado. Estas estrategias, en conjunto con una correcta evaluación, son consideradas por varios autores como las principales estrategias de enseñanza para la inclusión (Agut Horna, 2010; Cárdenas Leitón, 2011; Feliz Murias y Ricoy Lorenzo, 2002; Fernández Batanero, 2014; Flores Barrera et al., 2017; García Cedillo, 2010; Lledó Carreres, 2012; Marchesi, Durán, Giné y Hernández Izquierdo, 2009).

Las personas docentes en formación mencionaron que la capacitación recibida en cuanto a estrategias conductuales, habilidades adaptativas y estrategias de enseñanza 
sistemática era escasa. Asimismo, consideraron que pocas veces reciben recursos y/o apoyo de otros profesionales para trabajar con alumnos con NEE en sus lugares de práctica. Esto es similar a otros estudios en los cuales se sugiere la renovación de estrategias de enseñanza debido a la poca preparación y la carencia de recursos/apoyo recibidos de otros profesionales para atender la diversidad (Lledó Carreres y Arnaiz Sánchez, 2010; Santos Arrieta, 2014).

Según la Escala de Autoeficacia para Implementar Prácticas Inclusivas, se obtuvo una media general de 3.22, la cual indica que las personas docentes en formación tienen una percepción positiva sobre su autoeficacia para implementar prácticas inclusivas (ver Tabla 2). En la primera dimensión eficacia para dar instrucciones inclusivas, mencionaron ser capaces de proporcionar explicaciones o ejemplos alternativos cuando el alumnado está confundido. De igual forma, consideran que tienen confianza para hacer que el alumnado trabaje en grupos colaborativos. La media más baja se relaciona con la confianza en diseñar tareas de aprendizaje individualizadas.

En la dimensión eficacia en la colaboración, las personas docentes en formación indican estar de acuerdo en su capacidad para hacer que los padres de familia se sientan cómodos cuando van a la escuela. La media más baja se encontró en el reactivo: "tengo confianza para informar sobre leyes y políticas relacionadas con la integración de alumnos con discapacidad a quienes no saben".

En la dimensión eficacia en el manejo del comportamiento se observan en general puntuaciones positivas debido a que la mayoría de las personas docentes en formación respondieron estar de acuerdo. Sin embargo, muestran cierta desconfianza para manejar al alumnado que presenta problemas de agresividad.

Tabla 2

Puntuaciones medias de la Escala de Autoeficacia para Implementar Prácticas Inclusivas en personas docentes en formación del Sur de Sonora, México, 2018

\begin{tabular}{lcc}
\hline \multirow{2}{*}{ Dimensiones } & \multicolumn{2}{c}{$\begin{array}{c}\text { Docentes en } \\
\text { formación }\end{array}$} \\
\cline { 2 - 3 } & $\mathrm{M}$ & $\mathrm{DE}$ \\
\hline 1. Eficacia para dar instrucciones inclusivas & 3.33 & 0.46 \\
2. Eficacia en la colaboración & 3.20 & 0.48 \\
3. Eficacia en el manejo del comportamiento & 3.14 & 0.43 \\
\hline Total & 3.22 & 0.41 \\
\hline
\end{tabular}

Fuente: Elaboración propia, 2019 
En general, se encontró una percepción positiva en la autoeficacia de las personas docentes en formación. Las actividades áulicas en las que consideran ser más eficaces son: el proporcionar explicaciones y/o ejemplos alternativos, habilidad para promover el trabajo grupal y capacidad para tratar con los padres de familia. En menor medida, se encuentra la confianza para diseñar tareas de aprendizaje individualizadas, confianza para manejar estudiantes físicamente agresivos y confianza para informar sobre leyes y políticas relacionadas con la integración de alumnos y alumnas con discapacidad.

Al respecto de los tres puntos anteriores, varios autores han señalado que el profesorado que cuenta con experiencia trabajando con estudiantes con discapacidad y que ha tenido capacitación o entrenamiento en educación inclusiva tiene mejores niveles de autoeficacia (Leyser, Zeiger y Romi, 2011; Loreman, Sharma y Forlin, 2013; Lynn Peebles, 2012).

Las revisiones de la literatura sobre autoeficacia hacia la educación inclusiva en el profesorado muestran un claro énfasis en la experiencia previa de trabajo con alumnado con NEE para poder desarrollar una alta autoeficacia. Por ello, los resultados positivos en este estudio pueden ser explicados en el sentido de que durante la formación docente en las escuelas normales de México se promueven jornadas de observación y práctica, en las cuales las personas docentes en formación ponen en práctica las habilidades docentes y estrategias adquiridas. Esto, aunado a la retroalimentación de las personas formadoras de docentes y al análisis de la propia práctica, lo cual favorece el desarrollo de profesionales de la educación y la adquisición de competencias para una metodología de enseñanza sólida (Chapa Chapa y Flores Fahara, 2015).

Así, con la finalidad de determinar si existen diferencias significativas entre las puntuaciones medias obtenidas por el estudiantado de escuela normal urbana y rural, se aplicó la prueba $\mathrm{T}$ de Student para muestras independientes. En el Cuestionario de Estrategias para Fortalecer el Aprendizaje se encontraron diferencias estadísticamente significativas entre los grupos en la escala total $\mathrm{t}(164)=2.37, \mathrm{p}<.05$, así como en las subescalas contexto de aprendizaje $\mathrm{t}(164)=2.75, \mathrm{p}<.05$ y estrategias de cooperación interinstitucional $\mathrm{t}(164)=3.88, \mathrm{p}<.05$, lo cual indica que las personas docentes en formación de escuela normal urbana consideran llevar a cabo más estrategias en estas subescalas que los de la escuela normal rural. Las magnitudes del tamaño del efecto revelaron efectos bajos en la escala total y en la subescala estrategias contexto de aprendizaje, mientras que en la 
subescala estrategias de cooperación interinstitucional el efecto es moderado. Según Cohen (1988), los efectos se clasifican como bajos de 0.20 a .049 , moderados 0.50 a 0.79 y altos de 0.80 en adelante (ver Tabla 3).

En la Escala de Autoeficacia para Implementar Prácticas Inclusivas no se encontraron diferentes estadísticamente significativas entre las personas docentes en formación de escuela urbana y rural.

Tabla 3

Comparaciones de puntuaciones medias de personas docentes en formación de escuela urbana y escuela rural del Sur de Sonora, México, 2018

\begin{tabular}{|c|c|c|c|c|c|c|c|c|c|c|c|}
\hline \multirow{2}{*}{ Instrumento } & \multirow{2}{*}{ Dimensiones } & \multicolumn{3}{|c|}{ Urbana } & \multicolumn{3}{|c|}{ Rural } & \multirow[t]{2}{*}{$g l$} & \multirow[t]{2}{*}{$t$} & \multirow[t]{2}{*}{$p$} & \multirow{2}{*}{$\begin{array}{c}d \\
\text { Cohen }\end{array}$} \\
\hline & & $M$ & $D E$ & $N$ & $M$ & $D E$ & $N$ & & & & \\
\hline \multirow{6}{*}{$\begin{array}{l}\text { Estrategias } \\
\text { para } \\
\text { fortalecer el } \\
\text { aprendizaje }\end{array}$} & $\begin{array}{ll}\text { Estrategias } & \text { de } \\
\text { contexto } & \text { de } \\
\text { anrendizaie } & \end{array}$ & 3,40 & 0,32 & 77 & 3,25 & 0,37 & 89 & 164 & 2,75 & 0,006 & 0.43 \\
\hline & $\begin{array}{l}\text { Estrategias de } \\
\text { enseñanza }\end{array}$ & 3,37 & 0,44 & 77 & 3,30 & 0,42 & 89 & 164 & 1,07 & 0,283 & 0.16 \\
\hline & $\begin{array}{l}\text { Estrategias del } \\
\text { comportamiento }\end{array}$ & 3,29 & 0,36 & 77 & 3,15 & 0,55 & 89 & 164 & 1,81 & 0,071 & 0.30 \\
\hline & $\begin{array}{l}\text { Estrategias de } \\
\text { cooperación } \\
\text { interinstitucional }\end{array}$ & 3,49 & 0,33 & 77 & 3,25 & 0,45 & 89 & 164 & 3,88 & 0,0001 & 0.60 \\
\hline & $\begin{array}{l}\text { Estrategias de } \\
\text { recursos para el } \\
\text { aprendizaje }\end{array}$ & 2,76 & 0,68 & 77 & 2,85 & 0,74 & 89 & 164 & $-0,80$ & 0,423 & 0.12 \\
\hline & Escala total & 3,33 & 0,27 & 77 & 3,20 & 0,39 & 89 & 164 & 2,37 & ,019 & 0.38 \\
\hline \multirow{4}{*}{$\begin{array}{l}\text { Escala de } \\
\text { Autoeficacia } \\
\text { para } \\
\text { Implementar } \\
\text { Prácticas } \\
\text { Inclusivas }\end{array}$} & $\begin{array}{l}\text { Eficacia para dar } \\
\text { instrucciones } \\
\text { inclusivas }\end{array}$ & 3,38 & 0,37 & 77 & 3,30 & 0,53 & 89 & 164 & 1,12 & ,264 & 0.16 \\
\hline & $\begin{array}{l}\text { Eficacia en la } \\
\text { colaboración }\end{array}$ & 3,22 & 0,43 & 77 & 3,18 & 0,51 & 89 & 164 &, 50 & ,615 & 0.08 \\
\hline & $\begin{array}{l}\text { Eficacia en el } \\
\text { manejo del } \\
\text { comportamiento }\end{array}$ & 3,13 & 0,34 & 77 & 3,14 & 0,50 & 89 & 164 &,- 26 & ,795 & 0.02 \\
\hline & Escala Total & 3,24 & 0,31 & 77 & 3,21 & 0,48 & 89 & 164 &, 52 & ,600 & 0.07 \\
\hline
\end{tabular}

Fuente: Elaboración propia, 2019

Rodolfo Tuirán (2009, citado en Martínez, 2009) comentó "cinco aspectos que afectan la formación docente en las escuelas normales: 1) falta de preparación de las personas formadoras de docentes, 2) la selección del alumnado, 3) la falta de equipo y mal estado de instalaciones, 4) el rezago en los planes de estudio, y 5) la baja calidad de los planes 
curriculares", lo cual puede ser la respuesta a los datos encontrados en la comparación, en donde las personas docentes en formación de escuela normal rural llevan a cabo menos estrategias educativas inclusivas.

Respecto a los resultados de la comparación entre la escuela normal urbana y rural, se puede mencionar que el INEE (2016, citado en Hernández, García Tinoco y Solera, 2016) informó que, pese a los avances en infraestructura de las escuelas normales, aún persisten problemas, ya que los programas de mejora propuestos no han logrado impactar en la calidad de la formación docente. Este problema se incrementa en las escuelas normales rurales, en donde no se les forma para manejar grupos multigrado, entre otros aspectos. Por ello, se recomienda reconocer la diversidad de cada escuela normal y buscar soluciones oportunas según sus condiciones, con el propósito de evitar desfases (Hernández et al., 2016).

El análisis de relación entre las variables de estudio se calculó con la correlación de Pearson. En la Tabla 4 se puede observar que las tres dimensiones de la Escala de Autoeficacia (eficacia para dar instrucciones inclusivas, eficacia en la colaboración, eficacia en el manejo del comportamiento) presentaron correlaciones significativas y positivas con cuatro de las cinco subescalas del cuestionario de Estrategias, exceptuando la subescala estrategias de recursos para el aprendizaje. Estas relaciones pueden considerarse como moderadas (Ritchey, 2002). 
Tabla 4

Correlaciones de estrategias educativas inclusivas y autoeficacia de personas docentes en formación del Sur de Sonora, México, 2018

\begin{tabular}{|c|c|c|c|c|c|c|c|c|c|c|}
\hline Dimensiones & $\begin{array}{l}\text { Instrucciones } \\
\text { inclusivas }\end{array}$ & Colaboración & $\begin{array}{c}\text { Manejo del } \\
\text { comportamiento }\end{array}$ & $\begin{array}{l}\text { Autoeficacia } \\
\text { escala total }\end{array}$ & $\begin{array}{c}\text { Contexto } \\
\text { de } \\
\text { aprendizaje }\end{array}$ & Enseñanza & Comportamiento & $\begin{array}{l}\text { Cooperación } \\
\text { interinstitucional }\end{array}$ & $\begin{array}{c}\text { Recursos } \\
\text { para el } \\
\text { aprendizaje }\end{array}$ & $\begin{array}{c}\text { Estrategias } \\
\text { escala } \\
\text { total }\end{array}$ \\
\hline $\begin{array}{l}\text { Instrucciones } \\
\text { inclusivas }\end{array}$ & - & & & & & & & & & \\
\hline Colaboración & $0,684^{*}$ & - & & & & & & & & \\
\hline $\begin{array}{c}\text { Manejo del } \\
\text { comportamiento }\end{array}$ & $0,747^{*}$ & $0,661^{*}$ & - & & & & & & & \\
\hline $\begin{array}{l}\text { Autoeficacia } \\
\text { escala total }\end{array}$ & $0,907^{*}$ & $0,880^{*}$ & $0,892^{*}$ & - & & & & & & \\
\hline $\begin{array}{l}\text { Contexto de } \\
\text { aprendizaje }\end{array}$ & $0,331^{\star *}$ & $0,465^{\star *}$ & $0,260^{* *}$ & $0,397^{* *}$ & - & & & & & \\
\hline Enseñanza & $0,390^{* *}$ & $0,368^{* *}$ & $0,343^{\star *}$ & $0,411^{\star *}$ & $0,489^{* *}$ & - & & & & \\
\hline $\begin{array}{l}\text { Cooperación } \\
\text { interinstitucional }\end{array}$ & $0,357^{* *}$ & $0,345^{\star *}$ & $0,296^{\star *}$ & $0,373^{* *}$ & $0,661^{* *}$ & $0,480^{\star *}$ & $0,632^{\star *}$ & - & & \\
\hline $\begin{array}{l}\text { Recursos para } \\
\text { el aprendizaje }\end{array}$ & 0,034 & 0,144 & $-0,011$ & 0,065 & $0,354^{*}$ & 0,170 & $0,392^{\star *}$ & $0,563^{\star *}$ & - & \\
\hline $\begin{array}{l}\text { Estrategias } \\
\text { escala total }\end{array}$ & $0,379 *$ & $0,441^{*}$ & $0,312^{*}$ & $0,425^{*}$ & $0,854^{* *}$ & 0,709 & $0,825^{\star *}$ & $0,859^{* *}$ & $0,618^{* *}$ & - \\
\hline
\end{tabular}

Nota: *La correlación es significativa al nivel 0,05 (bilateral). ${ }^{*}$ La correlación es significativa al nivel 0,01 (bilateral)

Fuente: Elaboración propia, 2019 
La autoeficacia correlacionó positivamente y de manera significativa con las estrategias, de manera que las personas docentes en formación, que presentan un mayor nivel de autoeficacia, llevan a cabo más estrategias educativas inclusivas. Este resultado coincide con la investigación de Tschanen-Moran y Woolfolk (2001, citado en Herrera Seda, Schade Yankovic y Pérez Salas, 2016), en donde se menciona que el profesorado que posee alta autoeficacia es propenso a ser más creativo y flexible al momento de probar nuevos métodos de enseñanza. De igual manera, desarrolla mejores planificaciones, es organizado y se encuentra más motivado en su labor diaria.

De manera similar, Garduño Estrada, Carrasco Pedraza y Raccanello (2010), así como Prieto Navarro (2016) mencionan que la autoeficacia influye en el pensamiento, en las reacciones emocionales y en las conductas que las personas deciden llevar a cabo. Por lo tanto, si las personas docentes en formación se sienten capaces para desarrollar estrategias inclusivas, esto se verá reflejado en la práctica ya que mientras más altas sean las creencias de autoeficacia más fuerte será el compromiso para desarrollar prácticas y estrategias inclusivas.

\section{Conclusiones}

Al considerar los resultados obtenidos y la teoría revisada, se puede concluir que las personas docentes en formación de una escuela normal urbana y de una escuela rural, en la Región Sur de Sonora-México, desarrollan estrategias educativas que atienden la diversidad, entre ellas: el trabajo colaborativo, enseñanza de las TIC y algunas estrategias de retroalimentación y evaluación. Además, consideran que el apoyo recibido en cuanto a recursos y profesional especializado es escaso.

Un aspecto importante que surge en el trabajo y que coincide con la teoría revisada y con estudios recientes es la necesidad de formación inicial y permanente del profesorado, especialmente con temas relacionados con la educación inclusiva y con la atención a la diversidad. Al respecto, varios autores han puesto de manifiesto que es necesario contar con personal docente en formación capacitado, enfocado en identificar necesidades, para posteriormente adaptar los procesos de enseñanza y aprendizaje del alumnado. La formación docente debe estar encaminada a innovar estrategias, mejorar la atención educativa y permitir espacios de reflexión de toda la comunidad escolar, con el fin de favorecer los procesos inclusivos (Flores Barrera et al., 2017; García Navarro, Massani 
Enríquez y Bermúdez López, 2016; Mellado Hernández, Chaucono Catrinao, Hueche Oñate y Aravena Kennigs, 2016; Pegalajar Palomino y Colmenero Ruiz, 2017; SEP, 2017).

En cuanto a la comparación por tipo de escuela, se encontró que las personas docentes en formación de la escuela normal urbana llevan a cabo más estrategias educativas inclusivas que los de la escuela normal rural. Como se comentó anteriormente, las escuelas normales rurales han pasado por conflictos e inconsistencias a lo largo del tiempo. Mungarro (2009) menciona que las escuelas normales rurales han permanecido en un ambiente radical y politizado en el cual se ha cuestionado sobre la formación de los docentes en esos centros. Sin embargo, Civera (2015) indica que el trabajo y las condiciones de cada escuela normal dependerán de la región particular en donde se encuentren.

En cuanto a la autoeficacia, las personas docentes en formación se sienten capaces de promover el trabajo colaborativo, la enseñanza de habilidades y el desarrollo integral del alumnado. La autoeficacia se relaciona positiva y significativamente con las estrategias inclusivas, por lo cual se puede decir que cuanto mayor sea la capacitación y preparación del profesorado en educación inclusiva mayor será la autoeficacia percibida y reflejada en el desarrollo de prácticas incluyentes.

Vélez (2013) menciona que las acciones que realiza el profesorado son guiadas por el componente afectivo y cognitivo. Por consiguiente, es importante que las personas docentes en formación adquieran preparación, herramientas, conocimientos, así como actitudes, motivación y espacios de práctica adecuados para poder emprender el rol que les corresponde en un contexto inclusivo (Acedo, 2011; Buenestado Fernández, 2015).

Haro Soler (2017) propone cinco actividades prácticas para promover la autoeficacia de las personas docentes en formación. Como primera actividad se propone realizar un análisis objetivo de las propias capacidades, reconociendo puntos fuertes y habilidades. En segundo lugar, se propone el trabajo en pares para identificar de igual manera los puntos fuertes de cada uno. En tercer lugar, será importante tomar conciencia de lo identificado de manera personal y de manera colaborativa. En cuarto lugar, se puede propiciar que las personas docentes en formación aprendan de la experiencia de egresados, y por último, será crucial el establecimiento de objetivos personales como incentivos motivacionales. En conjunto, las actividades mencionadas fomentarán el reconocimiento de las capacidades y fortalecerán la autoeficacia. 
Sería conveniente realizar estudios similares con docentes en formación de diferentes universidades públicas y privadas, para conocer las diferentes concepciones sobre educación inclusiva que presentan y/o su formación docente. De igual manera, sería importante realizar estudios en este tema con otros actores y personas participantes en el proceso educativo, como pueden ser los directivos, personal administrativo, padres de familia y estudiantado de diferentes carreras, de manera que se pueda valorar la temática desde diferentes perspectivas.

En este trabajo solo se indagaron las percepciones de docentes en formación de escuela normal urbana y rural sobre su práctica, por lo que se considera conveniente profundizar en este aspecto llevando a cabo investigaciones que identifiquen y analicen las prácticas pedagógicas inclusivas en mayor profundidad. Esto puede llevarse a cabo mediante técnicas de registro de observación y otros cuestionarios que indaguen sobre prácticas o estrategias inclusivas, además de estudiarse de manera longitudinal.

Se recomienda realizar estudios con metodología cualitativa, ya que proporcionan información amplia e interesante para analizar la temática, la cual permite la comprensión de los fenómenos y las percepciones de las personas. Además, se considera de gran importancia desarrollar programas de capacitación en educación inclusiva para personas formadoras de docentes y docentes en formación de escuelas normales urbanas y rurales, que propongan estrategias y herramientas para trabajar en entornos inclusivos, independientemente de la diversidad del alumnado.

Por último, los hallazgos de este estudio resultan valiosos, ya que permiten mostrar el interés de las personas docentes en formación por la educación inclusiva y los diferentes aspectos que conlleva. Además, permite conocer los vacíos en la temática y las necesidades de formación actuales en las escuelas normales. 


\section{Referencias}

Acedo, Clementina. (2011). La preparación de los docentes para la educación inclusiva. Perspectivas. http://www.ibe.unesco.org/fileadmin/user upload/Publications/Prospects/ProspectsEdit orials/prospects159eds.pdf

Adame Sirgado, Virginia. (2015). Actitudes del profesorado ante el alumnado con necesidades educativas especiales (Tesis de maestría). Universidad de Extremadura, Badajoz, $\quad$ España). Recuperada de http://dehesa.unex.es/bitstream/handle/10662/3263/TFGUEX 2015 Adame Sirgado. pdf?sequence $=1$ \&isAllowed $=y$

Agut Horna, Neus. (2010). La evaluación en un modelo de escuela inclusiva. Aula de Innovación educativa, (191), 42-44. Recuperado de https://studylib.es/doc/4657150/laevaluaci\%C3\%B3n-en-un-modelo-de-escuela-inclusiva

Backhoff Escudero, Eduardo y Pérez Moran, Juan Carlos (coords.). (2015). Segundo Estudio Internacional sobre la Enseñanza y el Aprendizaje (TALIS 2013): Resultados de México. México: INEE. Recuperado de https://www.oecd.org/edu/school/MexicoTALIS-2013 es.pdf

Bandura, Albert. (1977). Self-efficacy: Toward a unifying theory of behavioral change. Psychological Review, 84(2), 191. Recuperado de http://citeseerx.ist.psu.edu/viewdoc/download?doi=10.1.1.315.4567\&rep=rep1\&type=pd $\underline{f}$

Beltrán Lera, Jesús. (2004). Estrategias de aprendizaje en Psicología de la Educación y del desarrollo en la edad escolar. Madrid: Editorial CCS

Buenestado Fernández, Mariana. (2015). Predicción de las actitudes hacia la inclusión de alumnado con necesidades educativas especiales en futuros profesionales de la educación (Tesis de maestría). Universidad de Córdoba, Córdoba, España. Recuperada

de https://helvia.uco.es/xmlui/bitstream/handle/10396/12787/Buenestado\%20Fernandez.p $\underline{\mathrm{df} \text { ? sequence}=1 \& \text { isAllowed }=\mathrm{y}}$

Cárdenas Leitón, Helvetia. (2011). Referentes teóricos y metodológicos de los docentes en servicio con una perspectiva de inclusión educativa: creencias y prácticas. Revista Electrónica Actualidades Investigativas en Educación, 11(2), 1-38. Recuperado de https://revistas.ucr.ac.cr/index.php/aie/article/view/10201

Chapa Chapa, Mireya y Flores Fahara, Manuel. (2015). La formación inicial de profesores en las Escuelas Normales. Recuperado de https://www.researchgate.net/publication/308401169 La formacion inicial de profesor es en las Escuelas Normales 
Chiner Sanz, Esther. (2011). Las percepciones y actitudes del profesorado hacia la inclusión del alumnado con necesidades educativas especiales como indicadores del uso de prácticas educativas inclusivas en el aula (Tesis doctoral). Universidad de Alicante, Alicante, España. Recuperada de https://rua.ua.es/dspace/bitstream/10045/19467/1/Tesis Chiner.pdf

Civera, Alicia. (1 de marzo, 2015). Normales rurales: Historia mínima de olvido. NEXOS. Recuperado de https://www.nexos.com.mx/?p=24304

Cohen, Jacob. (1988). Statistical power analysis for the behavioral sciences. Hillsdale, NJ: Lawrence Earlbaum Associates.

Covarrubias Apablaza, Carmen y Mendoza Lira, Michelle. (2013). La teoría de la autoeficacia y el desempeño docente: el caso de Chile. Estudios hemisféricos y polares, 4(2), 107123. Recuperado de http://www.revistaestudioshemisfericosypolares.cl/articulos/048Covarrubias\%20Lira-Autoeficacia\%20Desempeno\%20Docente\%20Chile.pdf

Darretxe Urrotxi, Leire; Goikoetxea Piedrola, Javier y Fernández González, Almudena. (2013). Análisis de prácticas inclusivas y exclusoras en dos centros educativos del país vasco. Revista Electrónica Actualidades Investigativas en Educación, 13(2), 1-30. [pdf]. Recuperado de http://www.redalyc.org/articulo.oa?id=44727049005 Doi: $\underline{10.15517 / a i e . v 13 i 2.11734}$

Durán Gisbert, David y Giné Giné, Climent. (2011). La formación del profesorado para la educación inclusiva: Un proceso de desarrollo profesional y de mejora de los centros para atender la diversidad. Revista Latinoamericana de educación inclusiva, 5(2). [pdf]. Recuperado de http://www.rinace.net/rlei/numeros/vol5-num2/art8 htm.html

Enríquez Félix, Misael. (2015). Paradigmas y prácticas en la Escuela Normal Rural. Revista de Educación y Cultura: La Escuela Normal, retos y perspectivas de la formación docente, (91), 14-17. Recuperado de https://educacionyculturaaz.com/la-escuelanormal-retos-y-perspectivas-de-la-formacion-docente/

Feliz Murias, Tiberio y Ricoy Lorenzo, María Carmen. (2002). La atención a la diversidad en el aula: Estrategias y recursos. Necesidades educativas especiales e intervención psicopedagógica, $1,123-162 . \quad$ Recuperado de https://www.academia.edu/842001/LA ATENCI\%C3\%93N A LA DIVERSIDAD EN E L AULA ESTRATEGIAS Y RECURSOS?auto=download

Fernández Batanero, José María y Hernández Fernández, Antonio. (2013). Liderazgo directivo e inclusión educativa: Estudio de casos. Perfiles educativos, 35(142), 27-41. Recuperado de http://www.scielo.org.mx/scielo.php?script=sci arttext\&pid=S0185$\underline{26982013000400003}$

Fernández Batanero, José María. (2013). Competencias docentes y educación inclusiva. Revista Electrónica de Investigación Educativa, 15(2), 82-99. [pdf]. Recuperado de http://redie.uabc.mx/vol15no2/contenido-fdzbatanero.html 
Fernández Batanero, José María. (2014). Un currículo para la diversidad. Madrid: Síntesis.

Flores Barrera, Vasthi Jocabed y García Cedillo, Ismael. (2016). Apoyos que reciben estudiantes de secundaria con discapacidad en escuelas regulares: ¿Corresponden a lo que dicen las leyes? Educación, 40(2). Recuperado de http://dx.doi.org/10.15517/revedu.v40i2.15851

Flores Barrera, Vasthi Jocabed; García Cedillo, Ismael y Romero Contreras, Silvia. (2017). Identificación de prácticas inclusivas de docentes formadores de docentes. Liberabit, 23(1), 39-56. doi: 10.24265/liberabit.2017.v23n1.03

Flores, Lirio y Villardón, Lourdes. (2015). Actitudes hacia la inclusión educativa de futuros maestros de inglés. Revista latinoamericana de educación inclusiva, 9(1), 61-73. Recuperado de https://dialnet.unirioja.es/descarga/articulo/5155477.pdf

Forlin, Chris; García Cedillo, Ismael; Romero Contreras, Silvia; Fletcher, Todd y Rodríguez Hernández, Roberto Javier. (2010). Inclusion in Mexico: ensuring supportive attitudes by newly graduated teachers. International Journal of Inclusive Education, 14(7), 723739. Recuperado https://www.tandfonline.com/doi/abs/10.1080/13603111003778569

García Cedillo, Ismael y Romero Contreras, Silvia. (2016). Avances de la integración educatival Educación inclusiva y la formación docente para la inclusión en México. México: Editorial Centro de Estudios Jurídicos y Sociales, A.C.

García Cedillo, Ismael. (2010). Educación inclusiva en Latinoamérica y el Caribe: El caso mexicano. Editorial Universitaria Potosina: México.

García Navarro, Xiomara; Massani Enríquez, Jorge Félix y Bermúdez López, Ivis Lourdes. (2016). La educación inclusiva en la formación de profesionales de la educación. Revista Conrado, 12(52), 6-10. Recuperado de https://conrado.ucf.edu.cu/index.php/conrado/article/download/273/272

Garduño Estrada, León; Carrasco Pedraza, Marco y Raccanello, Kristiano. (2010). Los formadores de docentes y la autoeficacia para la enseñanza en una muestra de escuelas normales en el estado de Puebla. Perfiles educativos, 32(127), 85-104. [pdf]. Recuperado de http://www.scielo.org.mx/pdf/peredu/v32n127/v32n127a5.pdf

George, Darren y Mallery, Paul. (2003). SPSS for Windows step by step: A simple guide and reference. 11.0 update. Boston: Allyn \& Bacon.

Haro Soler, María del Mar. (2017). ¿Cómo desarrollar la autoeficacia del estudiantado? Presentación y evaluación de una experiencia formativa en el aula de traducción. Revista Digital de Investigación en Docencia Universitaria, 11(2), 50-74. doi: http://dx.doi.org/10.19083/ridu.11.567

Hernández Sampieri, Roberto; Fernández Collado, Carlos y Baptista Lucio, Pilar. (2014). Metodología de la Investigación (6ta ed.). México: Mc Graw Hill. 
Hernández, Lilian; García Tinoco, Miguel y Solera, Claudia. (4 de diciembre, 2016). Normales rurales tocan fondo; SEP prepara plan para refundarlas. Excelsior. Recuperado de https://www.excelsior.com.mx/nacional/2016/01/17/1069328

Herrera Seda, Constanza; Schade Yankovic, Nieves y Pérez Salas, Claudia. (2016). El rol promotor de las concepciones, la autoeficacia y las prácticas de enseñanza de docentes universitarios en educación inclusiva. Revista Académica Discapacidad y Derechos, (1). Recuperado de https://www.researchgate.net/publication/320270987 El rol promotor de las concepc iones la autoeficacia y las practicas ensenanza de docentes universitarios en la inclusion educativa.

Infante, Marta. (2010). Desafíos a la formación docente: inclusión educativa. Estudios pedagógicos (Valdivia), 36(1), 287-297. Recuperado de http://www.scielo.cl/pdf/estped/v36n1/art16.pdf

Instituto Nacional para la Evaluación de la Educación [INEE]. (2015). Los docentes en México: Informe 2015. México: INEE. Recuperado de https://www.inee.edu.mx/wpcontent/uploads/2018/12/P11240.pdf

Lamorey, Suzanne y Wilcox, Marta. (2005). Early Interventionist Self-Efficacy Scale: a measure and its applications. Early Childhood Research Quarterly, 20(1), pp. 69-85. [pdf]. Recuperado https://www.sciencedirect.com/science/article/pii/S0885200605000049

Leyser, Yona; Zeiger, Tali y Romi, Shlomo. (2011). Changes in self-efficacy of prospective special and general education teachers: Implication for inclusive education. International Journal of Disability, Development and Education, 58(3), 241255. doi: 10.1080/1034912X.2011.598397

Lledó Carreres, Asunción y Arnaiz Sánchez, Pilar. (2010). Evaluación de las prácticas educativas del profesorado de los centros escolares: indicadores de mejora desde la educación inclusiva. Revista Iberoamericana sobre Calidad, Eficacia y Cambio en Educación, 8(5), 97-109. Recuperado de https://revistas.uam.es/index.php/reice/article/download/4729/5163

Lledó Carreres, Asunción. (2012). Luces y sombras en la educación especial hacia una educación inclusiva. Madrid: Editorial CCS.

Loreman, Tim; Sharma, Umesh y Forlin, Chris. (2013). Do Pre-service Teachers Feel Ready to Teach in Inclusive Classrooms? A Four Country Study of Teaching Self-efficacy. Australian Journal of Teacher Education, 38(1). Recuperado de http://files.eric.ed.gov/fulltext/EJ1008550.pdf

Lynn Peebles, Jodi. (2012). Examining the Impact of Direct Experience on Preservice Teachers' Self-Efficacy for Teaching in Inclusive Classrooms: a Quantitative Study (Doctoral dissertation). University of Calgary, Alberta, Canadá. Recuperado de http://theses.ucalgary.ca/bitstream/11023/301/4/ucalgary 2012 Peebles Jodi.pdf 
Marchesi, Álvaro; Durán, David; Giné, Climent y Hernández Izquierdo, Laura. (2009). Guía para la reflexión y valoración de prácticas inclusivas. Madrid: Organización de Estados Iberoamericanos para la educación, la ciencia y la cultura (OEI). Recuperado de https://www.fundacionmapfre.org/fundacion/es es/images/guia-practicas-inclusivasreflexion-valoracion tcm1069-421334.pdf

Martínez, Francisco. (2009). Los problemas de las escuelas normales. Reflexiones personales acerca de las noticias en los medios sobre la educación en México. Recuperado de http://educacioncafe.blogspot.com/2009/09/los-problemas-de-lasescuelas-normales.html

Medrano Camacho, Verónica; Ángeles Méndez, Eduardo y Morales Hernández, Miguel Ángel. (2017). La Educación Normal en México: elementos para su análisis. México: Instituto Nacional para la Evaluación de la Educación [INEE]. Recuperado de https://www.inee.edu.mx/wp-content/uploads/2019/01/P3B108.pdf

Mellado Hernández, María Elena; Chaucono Catrinao, Juan Carlos; Hueche Oñate, Marianela Clementina y Aravena Kennigs, Omar Andrés. (2017). Percepciones sobre la educación inclusiva del profesorado de una escuela con Programa de Integración Escolar. Revista Educación, 41(1), 1-14. Recuperado de https://revistas.ucr.ac.cr/index.php/educacion/article/view/21597/html

Mendoza, Zaria. (2015). Cambiarán plan de estudio de Escuelas Normales en Sonora. Uniradio Noticias. Recuperado de http://www.uniradionoticias.com/noticias/hermosillo/371495/cambiaran-plan-de-estudiode-escuelas-normales-en-sonora.html

Mitchell, David. (2014). What really Works in Special and Inclusive Education. New York: Routledge.

Mungarro, Jesús. (2009). La profesionalización de los docentes de las escuelas normales de Sonora. Ponencia presentada en el X Congreso Nacional de Investigación Educativa (COMIE) en 2009. Recuperado de http://www.comie.org.mx/congreso/memoriaelectronica/v10/pdf/area tematica 16/pone ncias/0518-F.pdf

Muntaner Guasp, Joan Jordi; Roselló Ramon, María Rosa y De la Iglesia Mayol, Begoña. (2016). Buenas prácticas en Educación Inclusiva. Educatio Siglo XXI, 34(1), 31-50. Recuperado de http://revistas.um.es/educatio/article/viewFile/252521/191611

Navarrete Cázales, Zaira. (2015). Formación de profesores en las Escuelas Normales de México. Siglo XX. Revista Historia de la Educación Latinoamericana, 17(25), 17-34. Recuperado http://revistas.uptc.edu.co/revistas/index.php/historia educacion latinamerican/article/vi ewFile/3805/3356 
Novo-Corti, Isabel; Muñoz-Cantero, Jesús-Miguel y Calvo-Babio, Nuria. (2015). Los futuros docentes y su actitud hacia la inclusión de personas con discapacidad. Una perspectiva de género. Anales de psicología, 31(1), 155-171. [pdf]. http://dx.doi.org/10.6018/analesps.31.1.163631

Organización de las Naciones Unidas [ONU]. (2007). El derecho a la educación de las personas con discapacidades: Informe del Relator Especial sobre el derecho a la educación Vernor Muñoz. Recuperado de http://www.right-to-education.org/sites/rightto-education.org/files/resource-

attachments/UNSR Derecho Educaci\%C3\%B3n Personas discapacidades 2007 ES .pdf

Ortega Tello, Sandra Elizabeth y Ortiz Ochoa, Jhanony Macarena. (2015). Prácticas inclusivas en el aula desde las percepciones de los estudiantes del séptimo año de educación general básica (Tesis de licenciatura). Universidad de Cuenca, Cuenca, Ecuador. Recuperada http://dspace.ucuenca.edu.ec/bitstream/123456789/21617/1/Tesis.pdf

Pegalajar Palomino, María del Carmen y Colmenero Ruiz, María de Jesús. (2017). Actitudes y formación docente hacia la inclusión en Educación Secundaria Obligatoria. Revista Electrónica de Investigación Educativa, 19(1), 84-97. Recuperado de https://redie.uabc.mx/redie/article/view/765/1498

Prieto Navarro, Leonor. (2016). Autoeficacia del profesor universitario: Eficacia percibida y práctica docente. México: Alfaomega.

Ramírez García, Antonia y Muñoz Fernández, María del Carmen. (2012). Prácticas inclusivas de los docentes en la convivencia escolar y en la organización y funcionamiento de los centros de educación primaria de la zona norte de Córdoba. Revista de Investigación Educativa, 30(1), 197-222. Recuperado

de http://revistas.um.es/rie/article/viewFile/116241/140161

Ritchey, Ferris. (2002). Estadística para las Ciencias Sociales: El potencial de la imaginación estadística. McGraw-Hill: México.

Romero Contreras, Silvia y García Cedillo, Ismael. (2013). Educación especial en México: desafíos de la educación inclusiva. Revista latinoamericana de educación inclusiva, 7(2), $\quad 77-91 . \quad$ [pdf]. $\quad$ Recuperado de https://dialnet.unirioja.es/descarga/articulo/4752911.pdf

Romero Contreras, Silvia; García Cedillo, Ismael; Forlin, Chris y Lomelí Hernández, Karla Abril. (2013). Preparing teachers for inclusion in Mexico: how effective is this process?. Journal of Education for Teaching, 39(5), 509-522. doi: $10.1080 / 02607476.2013 .836340$ 
Rubio-Moreno, Mireya; Castro-López, Gloria y Félix-Salazar, Valentín. (2015). Una aproximación a los procesos formativos del futuro docente de educación primaria. $R a$ Ximhai, 11(4), 381-402. Recuperado de http://www.redalyc.org/pdf/461/46142596028.pdf

Santos Arrieta, David. (2014). Percepción y Práctica Educativa Inclusiva en Docentes de Establecimientos Municipales de Monte Patria, IV región, Chile (Tesis de maestría). Universidad de Arte y Ciencias Sociales, Monte Patria, Chile. Recuperada de https://es.slideshare.net/la lagartija/percepcion-y-prctica-educativa-inclusiva-en-montepatria-david-santos-arrieta

Secretaría de Educación Pública [SEP]. (2017). Modelo Educativo para la Educación Obligatoria 2017. Recuperado de https://www.gob.mx/cms/uploads/attachment/file/207252/Modelo Educativo OK.pdf

Serrato Almendarez, Lilia Teresa y García Cedillo, Ismael. (2014). Evaluación de un programa de intervención para promover prácticas docentes inclusivas. Revista Electrónica Actualidades Investigativas en Educación, 14(3), 1-25. [pdf]. Recuperado de http://revista.inie.ucr.ac.cr/index.php/aie/article/viewFile/629/642

Sharma, Umesh; Loreman, Tim y Forlin, Chris. (2011). Measuring teacher efficacy to implement inclusive practices. Journal of Research in Special Educational Needs, 12(1), 12-21. doi: 10.1111/j.1471-3802.2011.01200.x

Tárraga Mínguez, Raúl; Peirats Chacón, José y Grau Rubio, Claudia. (2013). Creencias sobre la educación inclusiva de los futuros maestros y maestras de educación infantil y primaria. Comunicación presentada al X congreso Internacional y XXX Jornadas de Universidad y Educación Especial. Zaragoza, 2013. Recuperado de http://roderic.uv.es/handle/10550/40881

Vélez Latorre, Libia. (2013). La educación inclusiva en docentes en formación: Su evaluación a partir de la teoría de facetas. Revista FOLIOS, (37), 95-113. Recuperado de: http://www.scielo.org.co/pdf/folios/n37/n37a07.pdf 
Revista indizada en

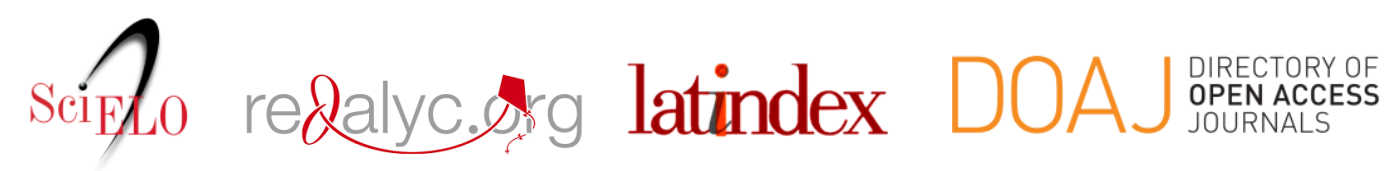

Distribuida en las bases de datos:

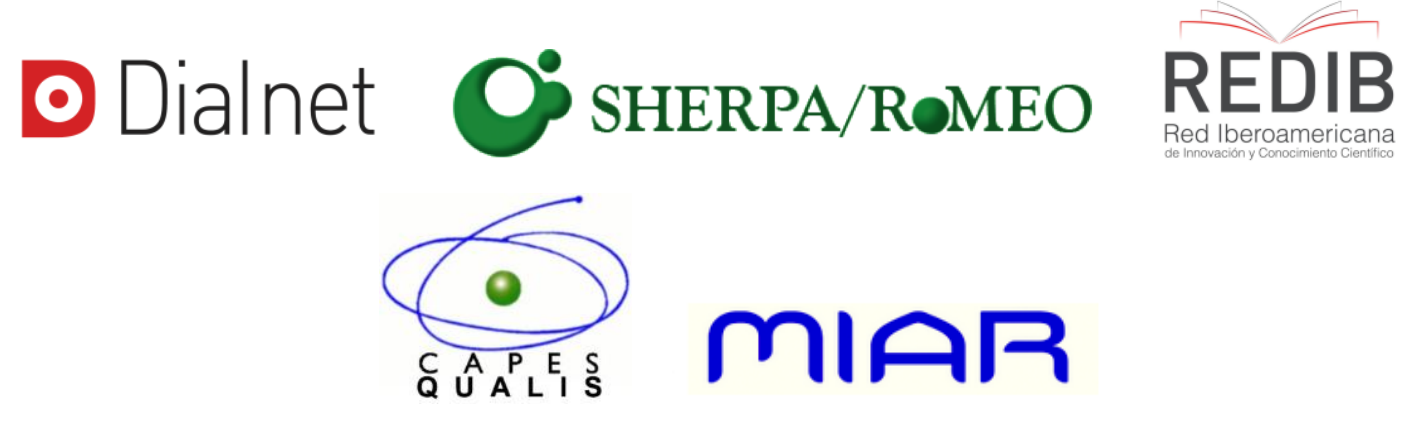

